\title{
Creencias de las mujeres víctimas de violencia que pertenecen a un estrato social alto en la Provincia de Tungurahua, Ecuador
}

\author{
Beliefs of women victims of violence who belong to a high social stratum in \\ the Province of Tungurahua, Ecuador
}

Shirley Katherine Argotti Pilataxi. ${ }^{1}$ \& Ana Elizabeth Jaramillo Zambrano. ${ }^{2}$

Recibido: 11-08-2021 / Revisado: 25 -08-2021 /Aceptado: 07-09-2021/ Publicado: 05-11-2021

\begin{abstract}
DOI: https://doi.org/10.33262/concienciadigital.v4i4.1.1929

Introduction. Violence against women has been a latent social phenomenon in Ecuador, there is a prevalence of $64.9 \%$ of emotional, physical, psychological violence and neglect towards women throughout their lives. Women victims of abuse from the upper socio economical class are not included in social, psychological or legal studies, because they do not file complaints, making it difficult for researchers to focus their attention on studying this issue. For this reason, there is a need to investigate the beliefs held by these women victims of violence and thus contribute to the state of the art. Target. identify personal, family and social beliefs about the violence to which women from the upper socioeconomic strata are subjected. Methodology. It is research of qualitative approach, of nonexperimental type and with descriptive and exploratory scope because it intends to search, specify properties, characteristics and important features through semi-structured interviews to analyze them and identify the beliefs of this population. We had a sample of 10 women between 25 and 60 years. Results. The results were obtained that women of high socioeconomic strata maintain beliefs based on different categories such as:

\footnotetext{
${ }^{1}$ Pontificia Universidad Católica del Ecuador Sede Ambato, Escuela de Psicología. Ambato, Ecuador. shirleykargottip@ pucesa.ec https://orcid.org/0000-0001-7304-4256

2 Pontificia Universidad Católica del Ecuador Sede Ambato, Escuela de Psicología. Ambato, Ecuador. ajaramillo@pucesa.edu.ec https://orcid.org/0000-0002-5754-598X
} 
personal, family and social, on the situation of violence of which they were at one time victims and that such beliefs, they continue to keep them in that condition of life. Conclusion. It is concluded that women victims of violence who belong to a high social stratum maintain personal, family and social beliefs.

Keywords: strata, beliefs, neglect, violence.

\section{Resumen}

Introducción. La violencia contra la mujer ha sido un fenómeno social latente en Ecuador, existe una prevalencia de un $64.9 \%$ de violencia emocional, física, psicológica y negligencia hacia la mujer a lo largo de su vida. Las mujeres víctimas de maltrato de la clase socioeconómico alta no figuran en estudios sociales, psicológicos o jurídicos, debido a que no ponen denuncias, dificultando que los investigadores centren su atención en estudiar esta problemática. Por esta razón, surge la necesidad de investigar cuáles son las creencias que mantienen estas mujeres víctimas de violencia y así contribuir al estado del arte. Objetivo. identificar las creencias personales, familiares y sociales acerca de la violencia de la cual son víctimas las mujeres de estrato socioeconómico alto de la provincia de Tungurahua. Metodología. Es una investigación de enfoque cualitativo, de tipo no experimental y con alcance descriptivo y exploratorio debido a que pretende buscar, especificar propiedades, características y rasgos importantes mediante entrevistas semiestructuradas para analizarlas y así identificar las creencias de esta población. Se contó con una muestra de 10 mujeres de entre 25 a 60 años. Resultados. Se obtuvo como resultados que las mujeres de estrato socioeconómico alto mantienen creencias basadas en diferentes categorías como: personales, familiares y sociales, sobre la situación de violencia de la cual fueron en algún momento víctimas y que dichas creencias, las siguen manteniendo en esa condición de vida. Conclusión. Se concluye que las mujeres víctimas de violencia que pertenecen a un estrato social alto, mantienen creencias personales, familiares y sociales.

Palabras claves: estratos, creencias, negligencia, violencia.

\section{Introducción}

La violencia contra las mujeres compone un grave problema sociocultural, considerando la complejidad del análisis social, el hecho de estudiar la realidad desde una perspectiva de género, no significa que se pueda despreciar otras variables de clasificación social, que marcan la diferente participación de las mujeres en instituciones sociales, económicas, políticas o religiosas, dada la configuración social actual en que las mujeres están sumergidas, comparten sus experiencias y obstáculos, no es difícil ver que existen muchos más aspectos por distinguir, como es el caso de esta investigación que se analiza desde un enfoque de las creencias instauradas en diferentes aspectos de vida como en lo personal, social y familiar (Lamas, 2013). En el Ecuador con la vigencia de la 
Constitución del 2008 la violencia contra las mujeres ha estado presente en la agenda pública nacional, en el desarrollo de políticas para la prevención y promoción mediante protocolos de atención que tienen como objetivo la erradicación de la violencia de género.

Para la investigación se apoya en datos proporcionados por el Instituto Nacional de Estadísticas y Censos [INEC], institución que, en la Ley Orgánica Integral para Prevenir y Erradicar la Violencia de género contra las Mujeres, establece en el "Art. 32" que:

Sin perjuicio de las facultades establecidas en la respectiva normativa vigente, tendrá las siguientes atribuciones: a) Levantar y proveer de información estadística al Registro Único de Violencia contra las Mujeres de acuerdo a los lineamientos y normativa emitidos por el ente rector de Sistema; b) Realizar encuestas especializadas en violencia a nivel nacional que aporten al Registro Único de Violencia contra las Mujeres, de conformidad con el Plan Nacional de Estadística; c) Las demás que establezca la normativa vigente (Asamblea Nacional República del Ecuador, 2018, p. 24).

Además de los derechos de las mujeres establecidos en La Convención Interamericana para Prevenir, Sancionar y Erradicar la Violencia contra la mujer (Convención Belém do Pará) en su artículo 1, define a la violencia contras las mujeres como "...cualquier acción o conducta, basada en su género, que cause muerte, daño o sufrimiento físico, sexual o psicológico a la mujer, tanto en el ámbito público como en el privado" (González, 2011). La violencia contra las mujeres, es un obstáculo para el desarrollo del país porque limita el ejercicio de derechos a la mitad de la población, amenaza su libertad y bienestar, también significa costos económicos para las familias, las comunidades, entre otros (Buvinic et al., 1999).

$\mathrm{Al}$ abordar de forma integral este fenómeno se evidencia que las causas de la violencia son multifactoriales. La encuesta nacional sobre relaciones familiares y violencia de género contra las mujeres en Ecuador, el INEC (2019) refiere que el $65 \%$ de cada 100 mujeres experimentan al menos un hecho de algún tipo de violencia en alguno de los distintos ámbitos a lo largo de su vida; madres, hijas, hermanas, víctimas que frente a una sociedad fragmentada por criterios convenientes, ya que, si la víctima es una infante, el rechazo es contra el agresor; no obstante, si la víctima es una mujer adulta los criterios morales ponen en tela de juicio el motivo de la agresión: el lugar en el que estuvo la víctima, la vestimenta que utilizaba, si el agresor era su pareja o un desconocido, el estatus social al que pertenece.

Además, el INEC (2019) menciona que a lo largo de la vida 43 de cada 100 mujeres sufren algún tipo de violencia por parte de su pareja de las cuales por encima del $80 \%$ no denunció; ahora bien, si en este contexto se plantea a la mujer dentro de un sistema socio familiar acaudalado, el miedo se transforma en un interrogante sobre la posición de poder que ejerce el maltratador que va desde mantener una imagen de felicidad que le otorga presuntamente su posición social y económica, patrones familiares heredados de madres a hijas, creencias irracionales condicionadas desde la niñez (Flores, 2015). Cuando una mujer es maltratada surgen varios hechos que influyen como los factores personales, 
familiares, sociales, económicos y culturales que colocan a las mujeres en situación de mayor riesgo frente a la violencia masculina (Camacho et al., 2014).

Por otro lado, el abuso de poder por parte del maltratador en el ciclo de la violencia se manifestó como fase de tensión, agresión y reconciliación. Sin embargo, la excepción es que estos agresores no tendían a pedir perdón después del evento violento, cuando se da la fase de reconciliación, sino que utilizaban los obsequios, regalos, viajes y otras cosas materiales como mecanismos para buscar la reconciliación con la cónyuge.

El estudio está desarrollado desde la teoría de la Representaciones Sociales (RS), que son entendidas como “... un corpus organizado de conocimientos y una de las actividades psíquicas gracias a las cuales los hombres hacen inteligible la realidad física y social, se integran en un grupo o en una relación cotidiana de intercambios" (Piña \& Yazmín, 2004). Desde esta perspectiva, se puede decir que la organización de conocimientos, tiene como resultado las creencias históricas, culturales, sociales y psicológicas que se han construido a lo largo de la historia de la humanidad y cuando se habla de violencia, dichas creencias, arraigan estas prácticas en el colectivo social, naturalizándolas y dando una visión de normalidad, llegando a ser aceptadas por la sociedad sin que se dé un cuestionamiento enfático sobre los actos violentos que se practican en la actualidad (Ariza, 2013).

La imposición del poder físico, económico y doméstico hacia la mujer desde esta teoría, se observar cómo el cuerpo de las féminas es el lugar en donde se plasman los simbolismos para ejercer violencia en cada una de ellas, creando una mirada objetiva y categorizada de creencias, valores, conductas, como si de un orden natural se tratara, enalteciendo su papel de madre, el rol en la crianza y cuidado de sus hijos, así también siendo la causante de desgracias, perversiones y calamidades (Álvaro y Fernández, 2006).

Sin duda, el patriarcado ha contribuido en la creación de esto simbolismos y creencias hacia la mujer, pues este sistema complejo de dominio, ha sido creado desde la historia tanto por hombres y mujeres, en donde el hombre ejerce el dominio sobre las mujeres y demás personas con las que forma su hogar, en donde ellas deben estar completamente privadas de derechos, influencia o recursos o poseer algún tipo de poder, lo que significa que ellos dominan las instituciones más importantes de la sociedad (Fernández y Duarte, 2006). Por tal motivo, el fenómeno de la violencia contra la mujer se ve influenciado por la sociedad patriarcal porque los roles y atributos tanto en los hombres como mujeres han dividido las diferencias biológicas. Estos mandatos han sido modelados por la familia, la escuela, iglesias, la calle, historias, cuentos, publicidad, la ideología hegemónica en la sociedad. Es por esto que se ve "normal" la subordinación y desventajas que día a día afrontan las mujeres; se cree que es lo habitual que, para mantener una familia unida, la mujer haga "sacrificios" como callar humillaciones, infidelidades, maltrato, para así ser catalogada con un título de buena o mala madre, esposa, hija o mujer (Camacho et al., 2014).

Segato (2003), habla sobre las estructuras elementales de la violencia hacia la mujer, donde se señala que las organizaciones de dominación patriarcal, el núcleo originario de violencia funciona de manera más o menos directa por medio de ataques sexuales, físicos, 
psicológicos o emocionales, donde la autora denomina violencia moral, que se encuentra interiorizados en la víctima, mujer, aceptando muchas veces el sistema de dominación. Esta violencia moral funciona en la vida cotidiana señalando no solo en el sitio que ocupa la mujer en el entorno del hogar, como hija y esposa, o fuera de ello como mujer trabajadora independiente, sino también al pensamiento mismo que les somete como poco racionales, abnegadas, sumisas, entre otras.

Por otro lado, hay que tomar en cuenta que, en la sociedad contemporánea, se escuchan diferentes tipos de expresiones, comentarios de las familias, bromas, proverbios, refranes, cualquier expresión coloquial sobre las mujeres, y que, a través de la repetición, los estereotipos (a manera de violencia) pueden permanecer profundamente arraigados en la mente del colectivo, perdurando en el tiempo y trasmitiéndose en las relaciones sociales. El Instituto Nacional de Desarrollo Social ([INDESOL], 2006), menciona que "Los estereotipos pueden convertirse en creencias, las que posiblemente lleguen a ser aceptadas como verdades absolutas por un grupo de personas. De ahí que las creencias podrían ser definidas como productos del pensamiento, de carácter subjetivo" (p. 61).

Para finalizar, la presente investigación tiene como objetivo evidenciar las creencias personales, familiares y sociales que tienen las mujeres del estrato socioeconómico alto, pues se cree que la violencia suele practicarse en los estratos socioeconómicos bajos, cuando la realidad es diferente a la percepción que tiene la colectividad, convirtiendo a esta creencia en una problemática social, ya que ellas no acceden a las estrategias de apoyo psicológicas, sociales y jurídicas, dejándose relegar y no permitiéndose obtener conocimiento para romper con los estereotipos sociales que tienen, y así liberase de sus maltratadores para que puedan tener una vida digna y libre de violencia.

\section{Metodología}

Es un estudio de enfoque cualitativo y no experimental porque se observa el fenómeno tal como se dio y no pretende manipular el hecho. El alcance de la investigación es exploratorio ya que el fenómeno de estudio gira en torno a la violencia a la mujer en estratos socioeconómicos altos; descriptivo por que se pretende buscar propiedades, características y rasgos importantes, analizando y describiendo las creencias de esta población; y explicativo al establecer las causas de los eventos, sucesos o fenómenos que se estudian (Hernández, 2018).

La población de este estudio está formada por 10 mujeres de 20 a 60 años de edad víctimas de violencia que pertenecen a un estrato socioeconómico alto. La técnica para seleccionar la muestra es Casos Tipo, el objetivo es la riqueza, profundidad y calidad de la información, no la cantidad ni la estandarización (Hernández, 2018).

Estas mujeres pertenecen a la provincia de Tungurahua que alguna vez, a lo largo de su vida, han sido víctimas de violencia de género (física, psicológica, sexual) y que han sido atendidas en el sistema de salud o de justicia. A sí también, es indispensable que sean mujeres que pertenezcan a un grupo socioeconómico alto, corroborado mediante aplicación de encuesta de estratificación socioeconómica. Para su participación, se 
empleó un consentimiento informado firmado por escrito a través del cual acepten ser parte en la investigación.

Mediante la aplicación presencial para una contención emocional adecuada de una entrevista semiestructurada con preguntas cerradas de tipo informativas, se recogió información sobre la edad, el nivel de estudios, estado civil, ocupación; y preguntas abiertas sobre la anamnesis: familiar, social y personal, la dinámica, frecuencia e importancia de sus relaciones afectivas en pareja en las que la mujer refiera la interpretación que tiene sobre las creencias que le llevaron a ser víctima de violencia, se estudia y se las categoriza mediante análisis de contenidos.

\section{Tabla 1}

Categorización sociodemográfica

\begin{tabular}{|c|c|c|c|c|c|c|c|}
\hline Código & Edad & Lugar de residencia & $\begin{array}{l}\text { Estado } \\
\text { civil }\end{array}$ & Religión & Escolaridad & Profesión & $\begin{array}{l}\text { No. de } \\
\text { hijos }\end{array}$ \\
\hline 1 & 29 & $\begin{array}{l}\text { Baños de Agua } \\
\text { Santa }\end{array}$ & Divorciada & Católica & Superior & $\begin{array}{l}\text { Psicóloga } \\
\text { clínica }\end{array}$ & 1 \\
\hline 2 & 38 & Ambato & Divorciada & $\begin{array}{l}\text { Cristian } \\
\text { a }\end{array}$ & Superior & Abogada & 1 \\
\hline 3 & 30 & Ambato & Casada & Católica & Superior & $\begin{array}{l}\text { Psicóloga } \\
\text { clínica }\end{array}$ & 2 \\
\hline 4 & 29 & Ambato & Soltera & $\begin{array}{l}\text { Cristian } \\
\text { a }\end{array}$ & Superior & Médico & 0 \\
\hline 5 & 57 & $\begin{array}{l}\text { Baños de Agua } \\
\text { Santa }\end{array}$ & Soltera & Católica & Bachiller & $\begin{array}{l}\text { Empresaria } \\
\text { hotelera }\end{array}$ & 1 \\
\hline 6 & 60 & Ambato & Casada & Católica & Superior & Abogada & 4 \\
\hline 7 & 31 & Ambato & Soltera & Católica & Superior & $\begin{array}{l}\text { Ingeniera } \\
\text { mecánica }\end{array}$ & 0 \\
\hline 8 & 29 & $\begin{array}{l}\text { Baños de Agua } \\
\text { Santa }\end{array}$ & Soltera & Católica & $\begin{array}{l}\text { Cuarto } \\
\text { Nivel }\end{array}$ & Turismo & 0 \\
\hline 9 & 30 & Quito & Soltera & Católica & Superior & $\begin{array}{l}\text { Ingeniera } \\
\text { electrónica y } \\
\text { redes de } \\
\text { información }\end{array}$ & 0 \\
\hline 10 & 28 & Ambato & Casada & Mormón & Secundaría & Ama de casa & 1 \\
\hline
\end{tabular}

En la tabla 1, se presenta la categorización sociodemográfica, se puede identificar que dentro de los rangos de edad propuestos entre 26-30 años se encuentran 6 mujeres; de entre 31-60 años, 4 mujeres; de la misma forma, en el lugar de residencia 6 mujeres viven en Ambato; en Baños viven 3 de ellas y en la ciudad de Quito una mujer. Por otro lado, en el estado civil, 3 mujeres son casadas, 2 divorciadas, 5 solteras. En cuando a la religión, las mujeres que son católicas son 7, cristianas 2, y de religión mormón 1. En lo que se refiere a escolaridad, una es bachiller, una terminó la secundaria, 7 de ellas cuentan con educación superior, y una mujer presenta cuarto nivel. Las profesiones con las que cuenta la población puesta en estudio fueron entre abogacía 2 mujeres, 2 de ellas son psicólogas clínicas, y una mujer para cada profesión que se menciona a continuación: ama de casa, ingeniera electrónica, redes de información, ingeniería mecánica, turismo, 
empresaria hotelera y médico. Por último, 4 mujeres no tienen hijos, 4 mujeres tienen un hijo, una mujer tiene 2 hijos y una mujer tiene 4 hijos.

Tabla 2

Categorización socioeconómica

\begin{tabular}{lll}
\hline Dimensiones & Subdimensiones & Pacientes \\
\hline Vivienda & Casa Villa & 3 \\
& Suite de lujo & 6 \\
Tecnología & Departamento & 1 \\
Bienes & Internet, celular, computadora, plan & 10 \\
& telefónico & \\
& Vehículo & 10 \\
Hábitos de consumo & Casa propia & 9 \\
& RUC & 8 \\
& Vestimenta en centros comerciales, Viajes & 10 \\
& a otro país, electrodomésticos de alta & \\
& gama, visita restaurantes exclusivos, asiste & \\
Nivel de escolaridad & a eventos sociales en lugares distinguidos & 10 \\
& Secundaria & 8 \\
& Superior & 4 \\
& Cuarto nivel & 1 \\
& PhD & 3 \\
& Acceso a educación internacional & \\
\hline
\end{tabular}

En la tabla 2 se puede identificar la categorización socioeconómica con el fin de evaluar que la población puesta en estudio se encuentran en un estrado socioeconómico alto, tomando en cuenta el código como el número de personas entrevistadas, dimensiones como vivienda, tecnología, bienes, hábitos de consumo, educación, economía en el hogar, tal como lo clasifica el INEC (2011) en la encuesta de estratificación socioeconómico el nivel A, es decir, que se encuentran en un nivel alto por las características observadas.

Desde el punto de las RA, una explicación en cuanto a los estratos sociales alto y bajo, las mujeres que presentan recursos económicos limitados suelen dirigirse a entidades gubernamentales públicas, para manifestar el maltrato por el que están pasando y busca atención o asesoría frente al problema, mientras que las mujeres que se encuentran en un estrato social alto, poseen mayor facilidades para dirigirse directamente con los profesionales como abogados, psicólogo; estás mujeres por lo general se encuentran alejadas de las cifras públicas de la violencia doméstica. 


\section{Tabla 3}

Matriz de Categorías

\begin{tabular}{|c|c|c|c|c|}
\hline Objetivo General & Categorías & Subcategorías & \multicolumn{2}{|c|}{ Unidad de Análisis } \\
\hline \multirow{3}{*}{$\begin{array}{l}\text { Identificar las creencias } \\
\text { personales, familiares y } \\
\text { sociales acerca de la } \\
\text { violencia que tienen las } \\
\text { mujeres de estrato social } \\
\text { alto }\end{array}$} & $\begin{array}{l}\text { Creencias } \\
\text { Personales }\end{array}$ & $\begin{array}{l}\text { - Autopercepción } \\
\text { - Frente a una relación afectiva de } \\
\text { Pareja (Rol establecido en género, } \\
\text { machismo) } \\
\text { - Historia de la situación de violencia. } \\
\text { (violencia física, psicológica o } \\
\text { sexual) }\end{array}$ & $\begin{array}{l}\text { Población } \\
\text { estudio. }\end{array}$ & de \\
\hline & $\begin{array}{l}\text { Creencias } \\
\text { Familiares }\end{array}$ & $\begin{array}{l}\text { - Estructura familiar (Rol hija) } \\
\text { - Dinámica familiar (Rol asumido } \\
\text { madre) }\end{array}$ & $\begin{array}{l}\text { Población } \\
\text { estudio. }\end{array}$ & de \\
\hline & $\begin{array}{l}\text { Creencias } \\
\text { Sociales }\end{array}$ & $\begin{array}{l}\text { - Comunidad. (Rol social) } \\
\text { - Religioso (Rol abnegación) } \\
\text { - Amistad. (estatus Social) } \\
\text { - Grupos de pertenencia. }\end{array}$ & $\begin{array}{l}\text { Población } \\
\text { estudio. }\end{array}$ & \\
\hline
\end{tabular}

\section{Resultados}

Luego del análisis de contenidos generados a partir de las entrevistas semiestructuradas sobre las creencias que las mujeres de estrato socioeconómico alto mantienen sobre la situación de violencia de la cual fueron en algún momento de su vida víctimas, se obtuvo resultados desde diferentes categorías como: personales, donde se identifica la autopercepción, la relación con la pareja, la historia de la situación; por otro lado, en la categoría familiar se identifica la dinámica de la misma que a través del genograma se pudo detectar diferentes características y la categoría de las creencias sociales con el fin de identificar el rol social, rol abnegación, estatus social y grupos de pertenencia.

\section{Tabla 4}

Análisis de las creencias personales que tienen las mujeres de estrato social alto víctimas de violencia

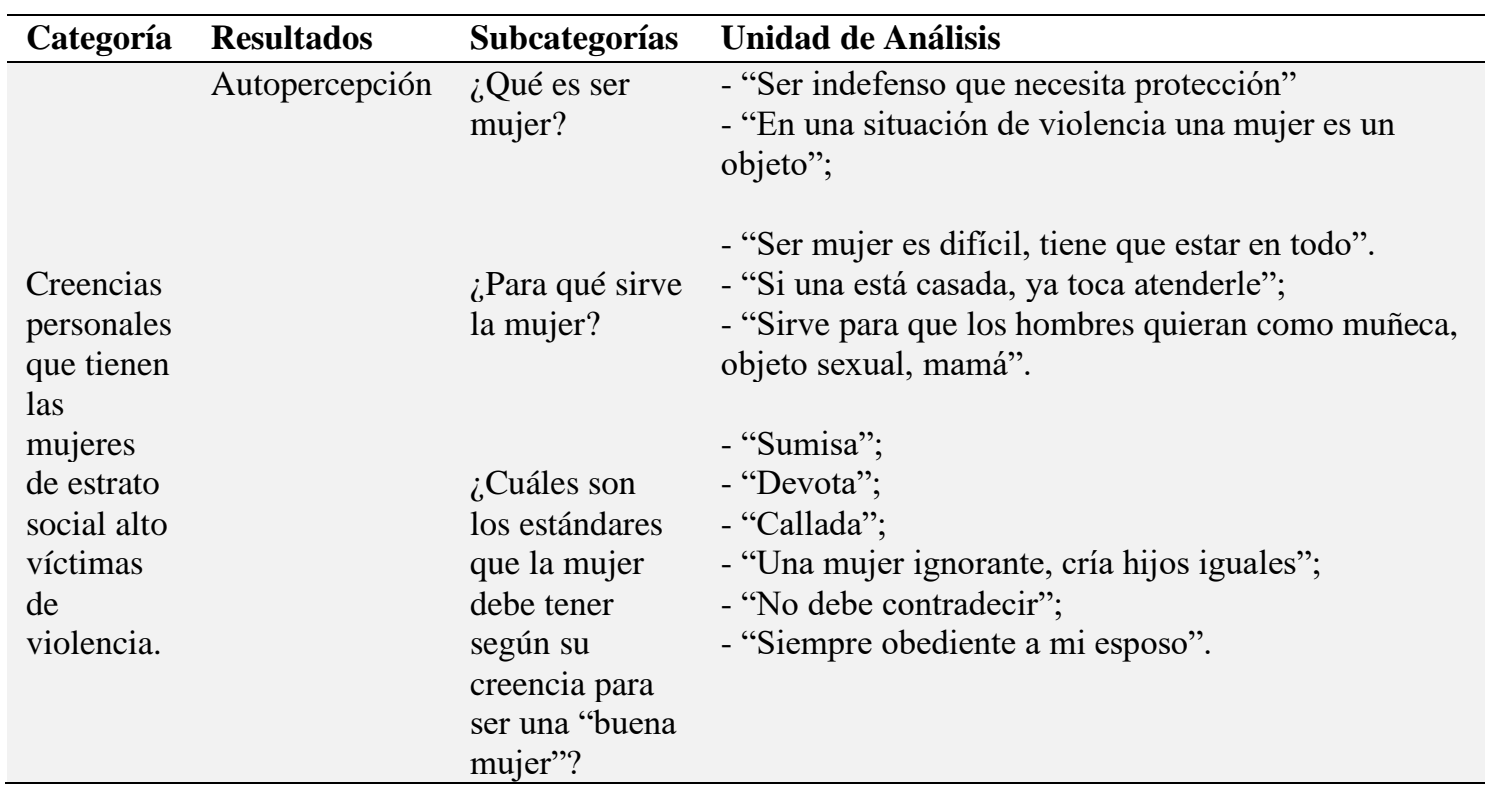




\section{Tabla 4}

Análisis de las creencias personales que tienen las mujeres de estrato social alto víctimas de violencia (continuación)

\begin{tabular}{|c|c|c|c|}
\hline Categoría & Resultados & Subcategorías & Unidad de Análisis \\
\hline \multirow[t]{2}{*}{$\begin{array}{l}\text { Creencias } \\
\text { personales } \\
\text { que tienen } \\
\text { las } \\
\text { mujeres } \\
\text { de estrato } \\
\text { social alto } \\
\text { víctimas } \\
\text { de } \\
\text { violencia. }\end{array}$} & $\begin{array}{l}\text { Frente a una } \\
\text { relación } \\
\text { afectiva de } \\
\text { Pareja }\end{array}$ & $\begin{array}{l}\text { Describa la } \\
\text { relación } \\
\text { afectiva más } \\
\text { relevante en su } \\
\text { vida según su } \\
\text { criterio: (edad, } \\
\text { tiempo, } \\
\text { dinámica de la } \\
\text { relación.) }\end{array}$ & $\begin{array}{l}\text { - "Sufrimiento, humillación, porque aguanté muchas } \\
\text { cosas, que no podía soportar"; } \\
\text { - "Era agresivo, pero me quedé porque no podía } \\
\text { avisar a mis padres"; } \\
\text { - "Una relación basada en el luchar". } \\
\text { - "Gracias a mi actual pareja logré superar mi } \\
\text { dependencia"; } \\
\text { - "Que te dejen ser"; } \\
\text { - "Si hay respeto en la relación va a durar mucho"; } \\
\text { - "Un vínculo fuerte para crecer"; } \\
\text { - "No sé, porque no lo he sentido"; } \\
\text { - "Ser protegida y contar con un respaldo". }\end{array}$ \\
\hline & $\begin{array}{l}\text { Historia de la } \\
\text { situación de } \\
\text { violencia }\end{array}$ & $\begin{array}{l}\text { ¿Es normal } \\
\text { para ti la } \\
\text { violencia en } \\
\text { una pareja, por } \\
\text { qué? }\end{array}$ & $\begin{array}{l}\text { - "No tener dinero propio"; } \\
\text { - "Hacer todo con permiso del esposo"; } \\
\text { - "Sentir que te odias a ti mismo"; } \\
\text { - "Fata de amor propio" } \\
\text { - "Te baja la autoestima"; } \\
\text { - "Sentirse inferior a él" } \\
\text { - "Ser violentada es no sentir, no pensar, no existir". } \\
\text { - "Me dijo que estoy gorda"; "amargada"; } \\
\text { - "Me despreció por mi baja remuneración"; } \\
\text { - "Debía cambiar mi forma de vestir"; } \\
\text { - "Me gritó porque no arregle la casa"; } \\
\text { - "Me golpeó, por no avisar que tomé un dinero". } \\
\text { - "Sí porque todos me decían que era normal, porque } \\
\text { su trabajo es estresante"; } \\
\text { - "Sí porque no somos suficientemente valientes para } \\
\text { cortar el problema"; } \\
\text { - "Sí porque es normal, para mantener una buena } \\
\text { imagen con los demás"; } \\
\text { - "Sí porque pensaba que la infidelidad se podía } \\
\text { soportar". }\end{array}$ \\
\hline
\end{tabular}

Desde el punto de vista de las RS, en cuanto a la categoría de autopercepción Simone de Beauvoir 1999, citado en Osborne y Molina (2008), menciona: "no se nace mujer, se llega a serlo" (p. 150). Es decir, no se nace siendo sensible, modesta, sumisa, afectuosa, devota, callada, no se nace con los atributos que posee la mujer, más bien, lo que se denomina feminidad o masculinidad son conductas que se obtienen, la adquisición de características que corresponden al género es un proceso cultural que se asimilan desde la interacción social con las personas que conforman el contexto próximo de la mujer. En el caso de las mujeres entrevistadas, la adquisición implica una negación de su trascendencia, al calificar la condición femenina, como una interpretación basada en la situación y no como consecuencia de la biología. 
De igual forma, con respecto a las creencias manifestadas por las mujeres sobre cuáles son los estándares que la mujer debe tener según su creencia para ser una "buena mujer", se asume bajo la perspectiva de Cobo (2005), en cuanto a la feminidad, tanto hombres como mujeres participan en la RS de la superioridad moral de las mujeres conocida también como excelencia moral, debido a que las creencias "No debo contradecir"; "Siempre obediente a mi esposo", aparentemente la disposición con otros da la idea de ser "buenas"; sin embargo, esta excelencia moral desde las mujeres se origina desde todo aquello que subordina, por ejemplo su asignación en el entorno doméstico y la separación desde espacios políticos-públicos. El significado importante de este argumento es que la excelencia se basa en una especie de norma, que es el resultado de la jerarquía patriarcal general y se resume en el desempeño de las tareas de cuidados y la capacidad de la mujer para ser emocional y empática con los demás.

Frente a una relación afectiva de pareja, unas de las respuestas claves que las mujeres manifestaron: "sufrimiento, humillación, porque aguanté muchas cosas, que no podía soportar"; "era agresivo, pero me quedé, porque no podía avisar a mis padres" las respuestas pueden relacionarse en funciones de las RS como aspectos de control. González (2011) menciona que las relaciones de poder pueden ir modificándose con el tiempo, alcanzando mayor nivel y convertirse en algo peligroso, y pueden ser invisibilizados por lo que es necesario develarlos. En este caso, las representaciones por parte de las mujeres se determina las creencias instauradas en base al domino que han establecido los hombres sobre ellas para "soportar" o "aguantar" cualquier clase de situaciones de pareja. En cuanto a la creencia de una mujer que acentúa sobre la descripción de la relación más relevante, respondió: "Una relación basada en el luchar". Frente a esto, Bourdieu y Passeron (1996), advierten que "Todo poder de violencia simbólica, o sea, todo poder que logra imponer significados e imponerlas como legítimas disimulando las relaciones de fuerza en que se funda su propia fuerza, añade su fuerza propia, es decir, propiamente simbólica, a esas relaciones de fuerza" (p. 44).

El autor señala, una probabilidad de la intencionalidad de poder de forma oculta. Escondidas por otras relaciones de fuerza donde se sobreentiende que el campo de batalla no demanda situaciones de crueldad, más bien el campo de una guerra simbólica.

Desde la historia de situaciones de violencia de las mujeres, se pudo identificar diferentes creencias en cuando al significado para las mujeres al ser violentadas, entre otras preguntas. Se toma en cuenta en este apartado a Simone de Beauvoir quien compara los sucesos de opresión de las mujeres con otros grupos oprimidos, donde se menciona que siempre ha existido una situación histórica donde las mujeres se sujetan a un grupo, sin embargo, quienes han permanecido subordinadas a los hombres por muchos años, han sido las mujeres describiéndose como esencial, porque viven entre los varones, no logran tener conciencia de grupo, además, se encuentran unidas por un vínculo a sus opresores. Dicho en palabras propias de la autora "negarse a ser alteridad, rechaza la complicidad con los hombres sería para ellas renunciar a todas las ventajas que les puede procurar la alianza con el superior" (De Beauvoir, 1949, p. 55). 
Es decir, las mujeres aunque han sido oprimidas por la forma de pensamiento sobre la negación de poseer la libertad tanto económica como social o política, ha sido porque a lo largo de la historia se ha seguido un patrón incensario en cuanto a la opresión, al igual que el esclavo no constituye un grupo con valores y con propia cultura, entonces la liberación por ende será diferente.

En cuanto a la pregunta ¿Es normal para ti la violencia en una pareja, por qué?, una de las respuestas fueron "Sí porque todos me decían que era normal, porque su trabajo es estresante"; para este apartado se toma en cuenta a González (2011), en su investigación sobre las mujeres violentadas habla sobre cómo se origina la violencia, y esta es desde las "formas de representación del patriarcado, instalado y sustentado en patrones culturales que se transmiten con un modo socialmente aceptado de relacionarse" (p. 121).

Estas afirmaciones, dan a entender como la sociedad viene naturalizando la violencia y como va aceptando conforme a la cultura en la que los seres humanos se desenvuelven, es a partir de esto que se instauran rasgos de socialización tanto femenina como masculina que maneja por su parte.

\section{Tabla 5}

Análisis de las creencias familiares que tienen las mujeres de estrato social alto víctimas de violencia

\begin{tabular}{|c|c|c|c|}
\hline Categoría & Resultados & Subcategorías & Unidad de Análisis \\
\hline $\begin{array}{l}\text { Creencias familiares } \\
\text { que tienen las mujeres } \\
\text { de estrato social alto } \\
\text { víctimas de violencia. }\end{array}$ & $\begin{array}{l}\text { Dinámica } \\
\text { familiar } \\
\text { (Rol } \\
\text { asumido de } \\
\text { la madre) }\end{array}$ & $\begin{array}{l}\text { ¿Considera que en su } \\
\text { sistema familiar ha } \\
\text { habido situaciones de } \\
\text { violencia que han sido } \\
\text { aceptadas por usted } \\
\text { cómo normales? }\end{array}$ & $\begin{array}{l}\text { - "Si, infidelidad como forma de } \\
\text { comunicación" } \\
\text { - "Sí, relación afectiva pasada con } \\
\text { agresión física y verbal" } \\
\text { - "yo no tengo recuerdos bonitos } \\
\text { de mi infancia" } \\
\text { - "Mis padres me impone sus } \\
\text { creencias, él es bien católico y yo } \\
\text { cristiana" }\end{array}$ \\
\hline
\end{tabular}

La familia es una parte importante de la sociedad por ser el primer espacio donde los individuos se desarrollan, las personas reciben los primeros cuidados para su crecimiento adecuado, la familia se convierte en la célula primordial de la sociedad y debe recibir el respeto, apoyo y orientación para promover su función como tal. Como base del ejercicio realizado, se puede mencionar que las RS de familia se diferencian de acuerdo a la manera de cómo cada individuo lo percibe dentro de la dinámica familiar. Betancur (2018), menciona sobre el sentido común de la teoría de las RS, originado por las costumbres, pensamientos, creencias, normas, tradiciones que en este caso es implantado por la familia, la misma que tiene como fin el orientar, integrar, interpretar acciones. Las mujeres se caracterizan por pertenecer a un sistema familiar extenso estructurado, familias monoparentales construidas.

En cuanto a la dinámica familiar y el rol asumido por la madre, se puede detectar que ha existido situaciones de violencia que han sido aceptadas como normales, entre estas infidelidades, relaciones efectivas pasadas con agresión de tipo física y verbal, situaciones 
que con el tiempo las mujeres pueden recordar que no han tenido un ambiente agradable en su infancia; en este caso la forma de autoridad ejercido puede estar asociada a las prácticas sociales que pueden conservarse de generación en generación, es por esto que López (2002), menciona: "La violencia vivida por los progenitores en la infancia se constituye en un modelo que se tiende a repetir" (p. 43).

Es muy común encontrar esta práctica en los hogares, aunque cierta información sobre daños y consecuencias que transita en los medios de comunicación en cuanto a los daños y sucesos desencadenantes, estos pueden convertirse en prácticas que generan miedo, desconfianza e inseguridad. Así también López (2002), menciona: "la repetición de patrones transmitida de generación en generación, se relaciona con la representación cultural que tiene los integrantes de una familia" (p. 122).

Desde estas indicaciones y el discurso social como referente para los miembros de la familia, ciertos patrones se van moldeando como el comportamiento, estilos de relación, estereotipos y roles asignados.

Por otro lado Barudy (2001), define a las familias desde un punto de vista cultural como "conductas que permanecen constantes a través de las generaciones (...) de discursos y/o relatos que se trasmiten y se mantienen de generación en generación y que dan sentido y explican los acontecimientos y fenómenos de la vida familiar” (p. 44).

Es por esta razón que la relación entre los miembros de la familia se estructura partiendo desde un estilo jerárquico rígido reapareciendo la desigualdad relacionada con estereotipos poco flexibles en relación al género que se obtiene desde las representaciones sociales dadas en un entorno amplio, los mismos influyen en las ciertas creencias sobre lo femenino y masculino.

\section{Tabla 6}

Análisis de las creencias sociales que tienen las mujeres de estrato social alto víctimas de violencia

\begin{tabular}{|c|c|c|c|}
\hline Categoría & Resultados & Subcategorías & Unidad de Análisis \\
\hline & $\begin{array}{l}\text { Comunidad. (Rol } \\
\text { social) }\end{array}$ & $\begin{array}{l}\text { Grupos sociales que } \\
\text { pertenece } \\
\text { actualmente o } \\
\text { perteneció en algún } \\
\text { momento de su vida }\end{array}$ & $\begin{array}{l}\text { De las } 10 \text { pacientes, } 5 \text { mencionaron al } \\
\text { grupo social de amigos, } 2 \text { a un } \\
\text { voluntariado, y } 2 \text { a un club } \\
\text { (membresía) }\end{array}$ \\
\hline $\begin{array}{l}\text { Creencias } \\
\text { sociales que } \\
\text { tienen las } \\
\text { mujeres de } \\
\text { estrato social } \\
\text { alto víctimas de } \\
\text { violencia. }\end{array}$ & & $\begin{array}{l}\text { ¿Cuáles son las } \\
\text { creencias que este } \\
\text { grupo social, } \\
\text { pudieron fomentar el } \\
\text { mantenerse en una } \\
\text { situación de } \\
\text { violencia? }\end{array}$ & $\begin{array}{l}\text { - "Era una responsabilidad, un peso } \\
\text { por mantener la imagen de una pareja } \\
\text { feliz para evitar comentarios", } \\
\text { - "El qué dirán, el tener una imagen } \\
\text { que este matrimonio estaba bien", } \\
\text { - "Yo me fui adaptando al maltrato de } \\
\text { a poco", } \\
\text { - "El no tener rechazo, ni dañar la } \\
\text { reputación de mi pareja", } \\
\text { - "Apariencia social" }\end{array}$ \\
\hline
\end{tabular}




\section{Tabla 6}

Análisis de las creencias sociales que tienen las mujeres de estrato social alto víctimas

\begin{tabular}{|c|c|c|c|}
\hline Categoría & Resultados & Subcategorías & Unidad de Análisis \\
\hline \multirow[t]{2}{*}{$\begin{array}{l}\text { Creencias } \\
\text { sociales que } \\
\text { tienen las } \\
\text { mujeres de } \\
\text { estrato social } \\
\text { alto víctimas de } \\
\text { violencia. }\end{array}$} & $\begin{array}{l}\text { Religioso (Rol } \\
\text { abnegación) }\end{array}$ & $\begin{array}{l}\text { ¿Cuáles son las } \\
\text { creencias que } \\
\text { mantienen las } \\
\text { mujeres de posición } \\
\text { socioeconómica alta } \\
\text { con respecto al } \\
\text { maltrato del cual son } \\
\text { víctimas? }\end{array}$ & $\begin{array}{l}\text { "Yo era casada" } \\
\text { "Yo soy creyente y el matrimonio es } \\
\text { importante", } \\
\text { "Si una ya está casada toca atenderle". }\end{array}$ \\
\hline & $\begin{array}{l}\text { Amistad. (estatus } \\
\text { Social) }\end{array}$ & $\begin{array}{l}\text { ¿Cuáles son las } \\
\text { creencias que } \\
\text { mantienen las } \\
\text { mujeres de posición } \\
\text { socioeconómica alta } \\
\text { con respecto al } \\
\text { maltrato del cual son } \\
\text { víctimas? }\end{array}$ & $\begin{array}{l}\text { - "Es preferible sonreír antes que la } \\
\text { gente murmulle", } \\
\text { - "Mantener la situación de violencia } \\
\text { reservada para mí, ya que podría ser } \\
\text { motivo de vergüenza y criticado en } \\
\text { nuestro círculo social", } \\
\text { - "Se debe mantener al margen la } \\
\text { exposición de este tipo de } \\
\text { dificultades", } \\
\text { - "No expresa el maltrato porque es } \\
\text { posible que se limite sus ingresos, así } \\
\text { callan", } \\
\text { - "Aparentar, el qué dirán por el } \\
\text { apellido", } \\
\text { - "Es tu culpa, no debes quejarte } \\
\text { porque tienes mucho de lo que otras } \\
\text { mujeres quisiera tener", } \\
\text { - "No debo reclamar porque el trae } \\
\text { todo lo necesario a la casa", } \\
\text { - "Una mujer separada es mal vista por } \\
\text { los amigos y la familia". }\end{array}$ \\
\hline
\end{tabular}

Desde las bases de las RS las creencias que presentan las mujeres víctimas de violencia de un estrato socioeconómico alto manifiestan creencias como: "Era una responsabilidad, un peso por mantener la imagen de una pareja feliz para evitar comentarios"; "El qué dirán, el tener una imagen que este matrimonio estaba bien", existen evidencia de que las mujeres asocian la violencia de género con múltiples factores relacionados con las características de personalidad del agresor o con el entorno social en el que se desenvuelve, asumiendo el rol social como creencia.

De esta manera Álvarez (2006), menciona que porque todos los individuos al momento de establecer una relación social pone en juego una serie de fuerzas personales con la confianza en sí misma, conocimientos, experiencias, recursos económicos, entre otros, que van a ser manejadas para defender y hacer realidad los deseos e intereses, las dificultades detectadas es que muchas de las veces no son tomadas en cuenta para desarrollar o reconocer esas fuerzas, dentro del sistema patriarcal en el que vivimos, esas fuerzas están determinadas por el lugar social que se ocupa y el valor social asignado según el género: masculino o femenino. 
Por otro lado, dentro del rol abnegado se identifican creencias como: "Yo soy creyente y el matrimonio es importante" se identifica creencias religiosas, se da a través de la historia donde se ejerce el derecho del hombre al castigo de la mujer, contemplándose en las tradiciones religiosas tanto cristianas como musulmanas y el nivel alto de valoración de concebir el matrimonio por parte de las mujeres y en la sociedad que se desenvolvían, mientras que las tradiciones del poder patriarcal justificaban que las mujeres casadas estuviesen siempre sujetas a la voluntad de su esposo (Ariza, 2013). Es decir, las creencias religiosas son factores desencadenantes que dirigen la violencia, porque sustentan históricamente el machismo, el poder patriarcal y así la contemplación de las mujeres a vivir en el maltrato.

Por último, la categoría de estatus social se identifica diferentes creencias donde se posicionan a las mujeres en un proceso de objetivación dentro de los procesos fundamentales de las RS, que según Jodelet en 1984, citado por Villaroel (2007), la primera fase se adapta a la selección y descontextualización de la información conforme a las normativas manifestadas de una cultura en particular; es decir, que la información se puede filtrar y desconectar del campo específico que originó de forma que el grupo social puede adueñarse y construirlas en sucesos de su propio mundo, de modo que pueden ser manejadas, como se puede evidenciar las creencias de tipo social, que influyen en el pensamiento de las mujeres, el mantener la situación de violencia reservada para sí misma, ya que podría ser motivo de vergüenza y crítica en el círculo social en el que se desenvuelven, o a su vez, el de mantener el matrimonio a pensar de ser violentada sólo por lo que llegasen a pesaran y a decir la gente de su contexto actual.

\section{Conclusiones}

- Es un hecho que la violencia de género es mortal en la vida de millones de mujeres y limita el desarrollo de la calidad de vida de cada una de ellas. En este estudio se puede identificar las creencias de las mujeres violentadas en un estrato social alto, en diferentes aspectos tanto personales, familiares y sociales. En lugar de sentirse seguras, muchas de las veces la familia es uno de los factores predominantes para padecer de abuso físico, psicológico, sexual y económico. Desde el punto de vista de las creencias personales en una investigación sobre el enfoque de género y la violencia contra las mujeres, propuesto por Álvarez (2006), menciona que las mujeres al momento de establecer una relación social ponen en juego una serie de fuerzas personales con la confianza en sí misma, conocimientos, experiencias, recursos económicos, entre otros, que van a ser manejadas para defender y hacer realidad los deseos e intereses. Además, las dificultades detectadas es que muchas de las veces no son tomadas en cuenta para desarrollar o reconocer esas fuerzas, dentro del sistema patriarcal en el que vivimos esas fuerzas están determinadas por el lugar social que se ocupa y el valor social asignado según el género: masculino o femenino.

- De tal forma, desde la perspectiva de las creencias familiares, sociales y personales analizadas en esta investigación se puede identificar que la socialización como el aprendizaje, son factores que se relacionan directamente 
hacia la reproducción de la violencia contra la mujer porque depende de la educación y del contexto sociocultural en el que se desenvuelve (Cobo, 2005). Sin embargo, la sociedad está formada por individuos, y cuando se los coloca en el grupo del que dependen, se comprenderá mejor su vida. La existencia de los individuos no se explica por sí misma, es necesario mostrar la estructura social en la que se inscriben estos individuos para comprender su significado individual.

- Las sociedades no solo están estratificadas por la existencia de clases sociales, porque no solo están configuradas con grupos jerárquicos y asimétricos en términos de estatus social y uso de recursos. Además, el género, la raza, la cultura, la etnia u orientación sexual constituyen una forma de estratificación que conduce a la formación de grupos con subordinación social y / o cuestiones de marginación económica, política y cultural.

- Por otra parte dentro del aspecto familiar las creencias que presentan las mujeres violentadas, se pudo observar en este caso una implantación de creencias que ha venido siendo una réplica de comportamientos y pensamientos por parte de las familias, a partir de los discursos y representaciones sociales que sirve de referencia para el método de estudio, son pautas para comprender cómo roles que van asumiendo en el hogar, va moldeando estereotipos, estilos de relación, sabiendo que todo se obtiene como un aprendizaje que se adquiere desde los primeros pasos, desde el donde se origina el ser humano, es decir, desde el núcleo familiar. Como lo menciona González (2011), "los sistemas de creencias, aprendizajes, normas y reglas que fueron adquiridas en la infancia y que se acuñan en la actual cotidianidad familiar" (p. 123).

- Las restricciones dentro de la familia promueven la acumulación de tensiones internas y la falta de cuestionamiento crítico del sistema de creencias sobre el abuso y la violencia. Por otro lado Barudy (2001), afirma en su investigación que las dinámicas familiares se dan como una manera de organización, que a través de las creencias establecidas van creando modelos de conductas, ideas, estilos de relación, influenciadas por la ideología patriarcal y que comprende "el aprendizaje de la obediencia y la sumisión a la autoridad del hombre" (p. 117).

- De esta afirmación se puede inferir que el postulado patriarcal afirma la visión de que los hombres son superiores a las mujeres y la devaluación basada en la creencia de que las mujeres son perdedoras y los hombres son valorados autoridad y autonomía. De este modo, se evidencia en las mujeres entrevistadas que los estilos de relación trasmitidos de generación en generación se instalan en el mandato del núcleo familiar, el aprendiza y creencias pueden replicarse en las generaciones siendo el reflejo de las mujeres, aunque cabe mencionar que no perciben de una forma natural de relacionarse, porque mucha de ellas han estado en una búsqueda constante de salida, demostrando la exclusión del medio en el que se encontraban.

- Es menester mencionar que la invisibilizarían de la violencia hacia la mujer ocurre en un estrato socioeconómico alto puede obedecer a la naturalización del abuso del poder, que es otorgado por el orden machista o patriarcal, donde los hombres 
tienen dicho control o poder sobre el ejercicio de la violencia hacia las mujeres. Como afirma Bourdieu (1998), la dominación del hombre sobre la mujer, se fundamente en la forma de cómo se construye el mundo a través de la dicotomía y estructural desarrollo de comportamientos, sentimientos, pensamientos, emociones y relaciones entre individuos, se instaura en ellos la forma de dividirse sexualmente del trabajo, y por ende, en el desarrollo de hábitos distintivo, esto se encuentra vinculado a estereotipos sobre el pensar y actuar de las mujeres que les conmina a ocultar las agresiones del cual son objeto, y en algunos casos (tal vez la mayoría) dando testimonios falsos en la atención médica, evitando dar a conocer al sistema judicial el caso.

- El silencio y ocultamiento contribuye a alimentar la violencia en la que son víctimas, limita el accionar del profesional médico, psicológico y de la policía nacional poniendo en peligro la vida. No obstante, el marco ético y legal intimida al profesional a cargo del caso, dar el respectivo seguimiento médico y psicológico, con el fin de proteger los derechos de las pacientes y darles a conocer sobre la problemática, precautelar posibles ataques violentos o evidenciar los riesgos de su vida y la de sus hijos. En este último, es importante acotar que si los actos violentos son presenciados por menores de edad, el personal a cargo está en la obligación de denunciar ante a la autoridad competente.

- Como diferentes autores por el orden machista mencionan desde el punto de las $\mathrm{RS}$, una explicación en cuanto a los estratos socioeconómicos alto y bajo, las mujeres que presentan recursos económicos limitados suelen dirigirse a entidades gubernamentales públicas para denunciar el maltrato por el que están pasando y buscan atención o asesoría frente al problema; mientras que las mujeres que se encuentran en un estrato socioeconómico alto, poseen mayor facilidades para dirigirse directamente con los profesionales como abogados, psicólogo. Estas mujeres por lo general se encuentran alejadas de las cifras públicas de la violencia doméstica, debido a que no denuncian el maltrato del cual son víctimas por el miedo "al qué dirán".

- Así también, la dependencia económica de la cual pueden estar sujetas, se convierte en un factor de riesgo en la violencia contra ellas porque es un aspecto común que sucede en un estrato socioeconómico bajo y alto con diferente propósito, siendo que en el bajo aparece la necesidad de cumplir con las necesidades básicas de sus hijos o de los que les rodea, como la alimentación, vestimenta; mientras que, en el estrato alto, por cumplir con llenar expectativas acordes a su estilo de vida. Bourdieu (1998), desde su mirada de la violencia simbólica como el sometimiento de unos individuos sobre otros, por medio del proceso de socialización que logra establecer las relaciones de poder, se convierten en hábito que aparecen de modo inconsciente, en cuanto la mujer al mantener la creencia de que depender del esposo será la única vía o camino, en un entramado de relaciones caracterizadas por miedos, inseguridades o la alusión "dependencia" para la toma de decisiones, lo cual la debilita aún más y la hace creer que sólo no puede conseguir ser exitosa e independiente. 


\section{Referencias bibliográficas}

Álvarez, O. (2006). El enfoque de género y la violencia contra las mujeres: aproximación al análisis de los conceptos. Revista Venezolana de Estudios de la Mujer, 3(1). Recuperado de http://ve.scielo.org/scielo.php?script=sci_arttextypid=S131637012006000100003

Álvaro, J., \& Fernández, B. (2006). Representaciones sociales de la mujer. Athenea digital(9), 65-77. Recuperado de https://www.redalyc.org/pdf/537/53700905.pdf

Ariza Sosa, G. (2013). Las representaciones sociales de la violencia en las relaciones de pareja en Medellín en el siglo XXI. Revista CES Psicología, 6(1), 134-158. Recuperado file://C:/Users/HP/Downloads/representaciones\%20sociales2.pdf

Asamblea Nacional República del Ecuador. (2018). Ley para prevenir y erradicar la violencia contra las mujeres. Quito-Ecuador: Asamblea Nacional República del Ecuador. Recuperado de https://www.igualdad.gob.ec/wpcontent/uploads/downloads/2018/05/ley_prevenir_y_erradicar_violencia_mujere s.pdf

Barudy, J. (2001). El tratamiento de familias en donde se producen abusos y malos tratos infantiles. Mallorca-España. Recuperado de http://www.buentrato.cl/pdf/est_inv/violen/vp_barudy.pdf

Betancur, Y. (2018). Representaciones Sociales de familia desde una perspectiva del desarrollo humano. Colombia: Universidad Externado de Colombia. Recuperado de de https://core.ac.uk/download/pdf/217416792.pdf

Bourdieu, P. (1998). La dominación masculina. Barcelona: Anagrama. Recuperado de http://www.nomasviolenciacontramujeres.cl/wpcontent/uploads/2015/09/Bondiu-Pierre-la-dominacion-masculina.pdf

Bourdieu, P., \& Passeron, J. (1996). La reproducción, elementos para una teoría del sistema de enseñanza. Madrid: Editorial popular.

Buvinic, M., Morrison, A., \& Shifter, M. (1999). La violencia en las Américas: un marco de acción. Washington: Banco Interamericano de Desarrollo.

Camacho, G., Larrea, C., \& Mendoza, C. (2014). La violencia de género contra las mujeres en el Ecuador: Análisis de los resultados de la Encuesta Nacional sobre Relaciones Familiares y Violencia de Género contra las Mujeres. Quito: Cooperación Española a través de la Agencia Española. Recuperado de https://oig.cepal.org/sites/default/files/violencia_de_gnero_ecuador.pdf

Cobo, B. (2005). El género en las ciencias sociales. Cuadernos de trabajo social ,249258. 
De Beauvoir, S. (1949). El segundo sexo. Cátedra, Madrid.

Fernández, R., \& Duarte, A. (2006). Preceptos de la ideología patriarcal asignados al género femenino y masculino, y su retractación en el ocho cuentos utilizados en el tercer ciclo de la educación general básica del sistema educativo. Revista Educación, $\quad 30(2), \quad$ 145-162. Recuperado de https://www.redalyc.org/pdf/440/44030210.pdf

Flores , A. (2015). Miedos y creencias irracionales en mujeres víctimas de violencia conyugal. 1-14. Recuperado de http://www.scielo.org.bo/pdf/rap/v2n1/v2n1a4.pdf

González, E. (2011). Mujeres y violencia transgeneracional: mitos y creencias que naturalizan el maltrato en los sistemas familiares. Revista perspectivas(22), 119137.

Hernández, R. (2018). Metodología de la Investigación: las rutas cuantitativas, cualitativas y mixtas. México: México: McGraw-Hill Interamericana. Recuperado de http://dspace.udla.edu.ec/bitstream/33000/8134/1/UDLA-EC-TPC-201732.pdf

Instituto Nacional de Desarrollo Social [INDESOL]. (2006). Diagnóstico sobre las causas, efectos y expresiones de violencia contra las mujeres en los hogares de la microrregión Huasteca Centro del estado de San Luis Potosí. San Luis Potosí,: Instituto de las mujeres del Estado de san Luis Potosi.

Instituto Nacional de Estadísticas \& Censos [INEC]. (2019). Encuesta de violencia contra la mujer. Recuperado de https://www.ecuadorencifras.gob.ec//violencia-degenero/

Instituto Nacional de Estadísticas y Censos [INEC]. (2011). Encuesta de Estratificación del Nivel Socioeconómico NSE 2011. Ecuador: INEC. Recuperado de https://www.ecuadorencifras.gob.ec//documentos/webinec/Estadisticas_Sociales/Encuesta_Estratificacion_Nivel_Socioeconomico/111 220_NSE_Presentacion.pdf

Lamas, M. (2013). El género, la construcción cultural de la diferencia sexual. México: programa Universitario de Estudios de Género. Recuperado de https://www.legisver.gob.mx/equidadNotas/publicacionLXIII/El\%20genero.\%2 0La\%20construccion\%20cultural\%20de\%201a\%20diferencia\%20sexual.pdf

López Díaz, Y. (2002). ¿Por qué se maltrata al más íntimo? . Colombia: Universidad Nacional de Colombia.

Osborne, R., \& Molina, C. (2008). Evolución del concepto de género1 (Selección de textos de Beauvoir, Millet, Rubin y Butler). Empiria(15), 147-182. Recuperado de https://www.redalyc.org/pdf/2971/297124045007.pdf 
Piña, J., \& Yazmín, C. (2004). La teoría de las representaciones sociales, su uso en la investigación educativa en México. Perfiles educativos, 26(105-106). Recuperado de de http://www.scielo.org.mx/scielo.php?script=sci_arttextypid=S018526982004000100005

Segato, R. L. (2003). Las estructuras elementales de la violencia Ensayos sobre género entre la antropología, el psicoanálisis y los derechos humanos. Argentina: Universidad Nacional de Quilmes. Recuperado de file://C:/Users/HP/Downloads/RCIEM137.pdf

Villaroel , G. (2007). Las representaciones sociales: una nueva relación entre el individuo y a sociedad. Fermetun, 434-454. Recuperado de https://www.redalyc.org/pdf/705/70504911.pdf

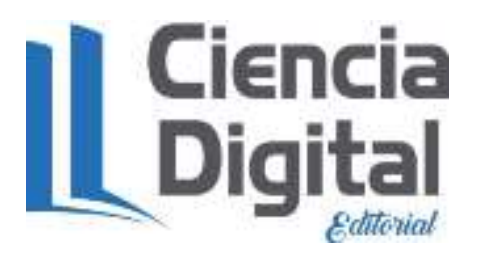




\section{PARA CITAR EL ARTÍCULO INDEXADO}

Argotti Pilataxi, S. K., \& Jaramillo Zambrano, A. E. (2021). Creencias de las mujeres víctimas de violencia que pertenecen a un estrato social alto en la Provincia de Tungurahua, Ecuador. ConcienciaDigital, 4(4.1), 111-130. https://doi.org/10.33262/concienciadigital.v4i4.1.1929

\section{LCiencia}

El artículo que se publica es de exclusiva responsabilidad de los autores y no necesariamente reflejan el pensamiento de la Revista Conciencia Digital.

El artículo queda en propiedad de la revista y, por tanto, su publicación parcial y/o total en otro medio tiene que ser autorizado por el director de la Revista Conciencia Digital.
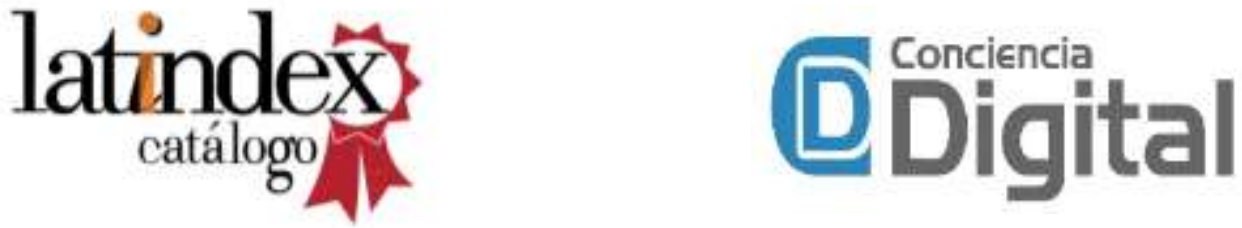